\title{
Synthetic lethal combinations of low-toxicity drugs for breast cancer identified in silico by genetic screens in yeast
}

\author{
Maximilian Marhold ${ }^{1}$, Erwin Tomasich ${ }^{1}$, Michael Schwarz ${ }^{2}$, Simon Udovica ${ }^{2}$, \\ Andreas Heinzel ${ }^{3}$, Paul Mayer ${ }^{3}$, Peter Horak ${ }^{4}$, Paul Perco ${ }^{3}$ and Michael Krainer ${ }^{1}$ \\ ${ }^{1}$ Department for Internal Medicine I-Oncology, Comprehensive Cancer Center, Medical University of Vienna, Vienna, Austria \\ ${ }^{2}$ Clinic of Internal Medicine I, Wilhelminenspital Wien, Vienna, Austria \\ ${ }^{3}$ Emergentec biodevelopment, Vienna, Austria \\ ${ }^{4}$ Department for Translational Oncology, German Cancer Research Institute (DKFZ), Heidelberg, Germany \\ Correspondence to: Michael Krainer, email: michael.krainer@meduniwien.ac.at \\ Keywords: synthetic lethality; breast cancer; drug combination; cancer; treatment
}

Received: February 14, 2017

Accepted: October 21, 2018

Published: November 20, 2018

Copyright: Marhold et al. This is an open-access article distributed under the terms of the Creative Commons Attribution License 3.0 (CC BY 3.0), which permits unrestricted use, distribution, and reproduction in any medium, provided the original author and source are credited.

\section{ABSTRACT}

In recent years, the concept of synthetic lethality, describing a cellular state where loss of two genes leads to a non-viable phenotype while loss of one gene can be compensated, has emerged as a novel strategy for cancer therapy. Various compounds targeting synthetic lethal pathways are either under clinical investigation or are already routinely used in multiple cancer entities such as breast cancer. Most of them target the well-described synthetic lethal interplay between PARP1 and BRCA1/2. In our study, we investigated, using an in silico methodological approach, clinically utilized drug combinations for breast cancer treatment, by correlating their known molecular targets with known homologous interaction partners that cause synthetic lethality in yeast. Further, by creating a machine-learning algorithm, we were able to suggest novel synthetic lethal drug combinations of low-toxicity drugs in breast cancer and showed their negative effects on cancer cell viability in vitro. Our findings foster the understanding of evolutionarily conserved synthetic lethality in breast cancer cells and might lead to new drug combinations with favorable toxicity profile in this entity.

\section{INTRODUCTION}

The concept of synthetic lethality describes a relationship between two genes, wherein loss of one gene can be compensated, but simultaneous loss-of-function of both genes results in a non-viable phenotype [1]. For synthetic lethal interactions identified in S. cerevisiae, C. elegans or Drosophila spp., potential conservation in the human genome was suggested and further proposed as suitable targets for precision cancer therapy [2-4].

The first predicted human synthetic lethality gene interactions that led to the development of approved therapeutics were those of Breast Cancer genes 1 and 2 (BRCA 1/2) and poly (ADP-ribose) polymerase 1 (PARP1). Tumor cells deficient in either BRCA gene were highly susceptible to PARP inhibition, whilst this was not the case for BRCA wildtype cells [5, 6]. Whether by inhibiting PARP1-supported single-strand repair (SSR) or by trapping PARP at the DNA damage site, PARP inhibitors induce DNA lesions that require homology directed repair (HDR) [7-9]. Since both BRCA genes play essential roles in HDR pathways in humans [8], loss-of-function of either BRCA1 or 2 sensitizes cells to PARP inhibitors.

During recent years, successful efforts have been undertaken to discover further, novel and clinically significant synthetic lethal gene combinations, by using molecular biology approaches such as genetic RNA interference or CRISPR/Cas libraries [10-12]. Furthermore, in silico approaches using machine learning and network properties were shown to be valuable tools in identifying novel genetic as well as chemico-genetic interactions causing synthetic lethality $[13,14]$. 
In this study, we used known negative genetic interactions in yeast to create a machine learning-based synthetic lethality predictor for human cancer cells. Based on novel synergies predicted in silico by our model, we were then able to verify the efficacy of the corresponding lowtoxicity treatment combinations for breast cancer in vitro.

\section{RESULTS}

\section{Creation of a systematic database of drug combinations in cancer therapy}

Exploring the website "http://clinicaltrials.gov" for "cancer" and limiting results for trials in phase III or IV created a set of 6,665 trials, of which 643 met our requirements of oncologic indication and pharmacological intervention. These trials contained 790 different drug combinations. An additional 121 combinations were found in clinical practice. Taken together, a total of 911 different drug combinations spanning 119 indications were found (Supplementary Figure 2). 150 individual drugs were identified, targeting 285 different genes. The excess of targets compared to drugs was due to certain drugs targeting multiple proteins. Most frequently occurring drugs were cyclophosphamide, etoposide, and doxorubicin. Summarized in classes, half of the drugs were found to be antimetabolites, alkylating agents, antimicrotubule agents or anthracyclines (data not shown).

Among the 119 different oncology treatment indications, breast cancer was the most frequent. As expected, this indication included the most drug combinations as well as the highest number of individual drugs (Table 1). The drug classes most abundant for breast cancer were antimicrotubule agents, antimetabolites, alkylating agents and anthracyclines (Figure 1A). A map showing drug combinations in breast cancer clinical trials was created and is illustrated in Figure 1B.

The drugs were then rearranged to form novel combinations, targeting synthetic lethal gene pairs as suggested by our in silico predictor based on a machinelearning algorithm. After filtering the resulting list for low toxicity combinations, the drug pairs celecoxib/zoledronic acid (ZOL/CEL) and olaparib/zoledronic acid (ZOL/OLA) were selected for further in vitro evaluation (Figure 2).

\section{Predicted synthetic lethality in breast cancer confirms highly efficient drug combinations already used in clinical routine}

Among drugs already used in clinical practice, the predictor identified six drug pairs potentially targeting gene pairs in a synthetic lethal manner. These six combinations consisted of bevacizumab, docetaxel, gemcitabine, paclitaxel, and trastuzumab (Table 2 and Figure 3).

\section{Predicted drug combinations significantly reduce viability of breast cancer cells in vitro}

When treated with the drug combinations suggested by our synthetic lethality predictor, breast cancer cell lines MDA-MB-468 and SKBR-3 exhibited decreased viability in a significant and synergistic manner. Both drug combinations showed a significant reduction in cell viability compared to single agent treatment in both cell lines (Figure 4). Surprisingly, the observed synergy was most pronounced at lowest concentrations used. Most noteably, the combination of olaparib and zoledronic acid showed a favourable combinational index at all concentrations and in both cancer cell lines studied.

In MCF12A cells derived from benign mammary epithelium, on the other hand, combination treatment with either ZOL/CEL or ZOL/OLA did not cause synergistic declines in cell viability, indicating cancer-specificity of the effects observed (Supplementary Figure 4C).

Compatible with our findings on cell viability, immunoblotting analyses substantiate the suggested disruption of antiapoptotic and proliferative signaling through Akt and Erk in breast cancer cells upon treatment with ZOL/CEL and ZOL/OLA (Figure 5B). Further, reductions in cell viability observed were shown to be caused in part by induction of apoptosis using AnnexinV/7-AAD stainings in both MDA-MB-468 and SKBR-3 cells (Supplementary Figure 3).

\section{Triple-negative breast cancer cells are highly susceptible to zoledronic acid treatment}

We observed a more than 100-fold difference of zoledronic acid-related cytotoxicity between the two cell lines studied, which lasted in repetition (Figure 4A). The MDA-MB-468 cell line derives from triple-negative breast cancer (TNBC) and strongly responded to zoledronic acid treatment, while Her2/neu overexpressing SKBR-3 cells did not respond in a similar manner. We were able to further confirm TNBC sensitivity towards zoledronic acid treatment using the HTB-26 cell line (Supplementary Figure 4A, 4B). To our knowledge, the observed effect of zoledronic acid on TNBC cell viability has not been described before in such significance.

\section{DISCUSSION}

In our work, we identified novel and targetable synthetic lethality partner genes in breast cancer using an in silico approach based on gene interactions in yeast and known molecular drug targets for human cancer. The advantage of such cross-species methodologies, which have led to discovery of various synthetic lethal partner genes $[15,16]$, lies in their reliability as well as costefficiency, when compared to large-scale genetic knockout 
Table 1: Oncologic entities and assigned drug combinations

\begin{tabular}{lccc}
\hline Disease term & $\begin{array}{c}\text { Drug } \\
\text { combinations }\end{array}$ & $\begin{array}{c}\text { Individual } \\
\text { drugs }\end{array}$ & $\begin{array}{c}\text { Drug } \\
\text { targets }\end{array}$ \\
\hline Breast Neoplasms & 145 & 64 & 70 \\
Carcinoma, Non-Small-Cell Lung & 98 & 58 & 84 \\
Leukemia, Myeloid, Acute & 76 & 43 & 83 \\
Lymphoma, Non-Hodgkin & 58 & 42 & 45 \\
Multiple Myeloma & 49 & 33 & 44 \\
Leukemia, Lymphocytic, Acute & 48 & 38 & 45 \\
Colorectal Neoplasms & 47 & 38 & 86 \\
Ovarian Neoplasms & 33 & 25 & 36 \\
Stomach Neoplasms & 29 & 26 & 37 \\
Pancreatic Neoplasms & 25 & 31 & 69 \\
Prostatic Neoplasms & 25 & 29 & 68 \\
Hodgkin Disease & 24 & 28 & 36 \\
Leukemia, Lymphocytic, Chronic & 17 & 16 & 29 \\
Melanoma & 17 & 23 & 30 \\
Esophageal Neoplasms & 17 & 18 & 25 \\
\hline
\end{tabular}

or knockdown experiments [13, 17-19]. However, due to evolutionary conservation, the found gene interactions might cause higher side effects when targeted, which could be seen as a disadvantage compared to largecomputational cancer-specific efforts [20]. Because of this excess toxicity concern, this study focused on drug combinations comprising of relatively non-cytotoxic treatment partners, such as celecoxib and zoledronic acid, and evaluated their biological activity when tested in vitro.

Zoledronic acid is known to inhibit bone degradation in osteoporosis and to prevent bone metastasis in breast cancer patients [21, 22]. It has also been shown to prolong survival in premenopausal women with endocrine-responsive early breast cancer $[23,24]$. Zoledronic acid belongs to the chemical group of nitrogenous bisphosphonates. One trait to this class of molecules is the blockade of the mevalonate pathway, which has been shown to be essential for prenylation and hence anchoring and activation of Ras at the cell membrane $[25,26]$. Consistent with this mechanism, nitrogen-containing bisphosphonates were shown to exhibit anti-tumor activity in vitro by inducing apoptosis or blocking invasion and spreading [27, 28]. Other targets of zoledronic acid according to the DrugBank database include geranylgeranyl pyrophosphate synthase (GGPS1) and hydroxylapatite [29, 30]. Zoledronic acid was further shown to reverse epithelial-to-mesenchymal transition (EMT) and inhibit self-renewal in TNBC [31]. Differential activity of zoledronic acid in the two cell lines studied may be explained through pathway addiction to EGFR and hence Ras activity in triple-negative breast cancer [32]. The potential beneficial effects are also reflected in a recent meta-analysis showing a trend for improved patient outcomes in women suffering from TNBC who had been treated with zoledronic acid [33].

Celecoxib targets cyclooxygenase 2 (COX-2), but - according to the DrugBank database used for creation our model - further affects six other targets such as 3-phosphoinositide-dependent kinase-1 (PDPK1), a known activator of Akt [34-36]. Besides, cytostatic effects of celecoxib combined with sorafenib have been observed in vitro in various cancer cell lines [37, 38]. Additionally, celecoxib was shown to benefit patients with familiar adenomatous polyposis (FAP) by significantly reducing numbers of colorectal polyps [39].

Olaparib is a PARP1 inhibitor, a drug class whose discovery and use is based on synthetic lethality $[5,6$, 40-42]. PARP1 detects and binds to DNA lesions, then produces poly(ADP-ribose) (PAR) chains, which function as a scaffold for the cellular DNA repair machinery [41]. Its inhibition has been shown to either cause deficient repair of single-strand breaks or to trap PARP at the damage site, thus creating an obstacle during the creation of the replication fork $[7,9,43]$.

We reason that the synthetic lethal synergisms predicted by our yeast-based in silico approach and tested in vitro, are caused by simultaneous disruption of two interdependent pathways: Zoledronic acid interferes with the activation of Ras by inhibiting its prenylation while celecoxib blocks activation of Akt by inhibiting PDPK1. As generally accepted in the scientific community, both Ras and Akt pose crucial proliferative and antiapoptotic stimuli that cause cell death when successfully antagonized (Figure 5A). Olaparib, blocking nuclear changes following 

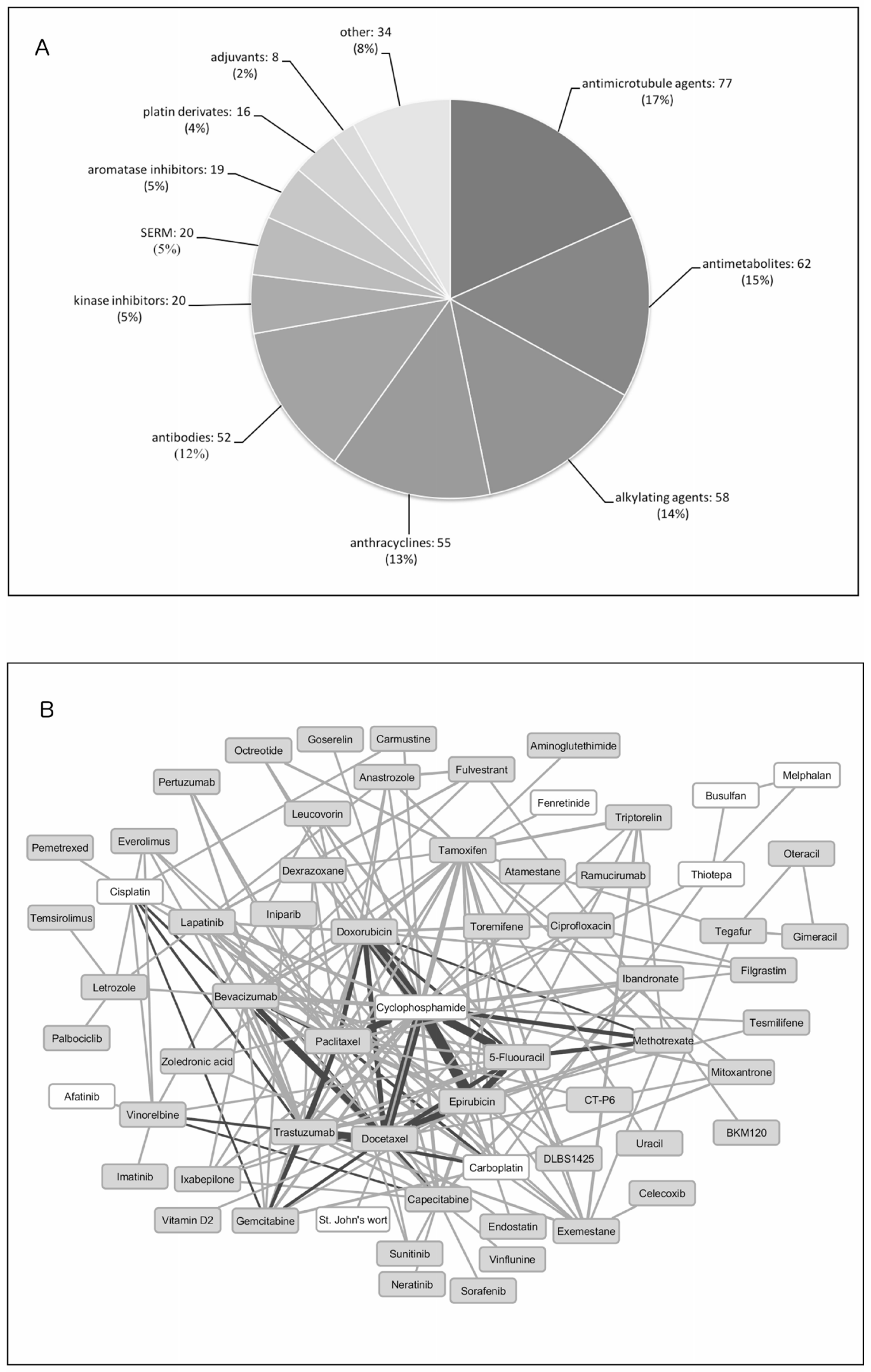

Figure 1: Drugs found in clinical practice and clinical trials for breast cancer. (A) Pie chart representing drugs found in clinical practice for breast cancer clustered according to their mechanism of action. (B) Drug-drug combinations extracted from phase III and IV breast cancer clinical trials. White background drugs have no specific target protein. Dark lines represent drug combinations also used in clinical practice. Line width correlates with frequency in which drugs are combined. 
a proliferative signal, might cause cellular vulnerability in case of disruption of a second mitogenic signaling effector, such as Ras. In addition, a potential relationship between Ras and PARP was suggested by Liu and colleagues, who found PARylation of H-Ras to be important for stabilization of H-Ras and normal cell cycle progression [44].

Both cell lines used in our study are known to express wild-type BRCA1, which explains the moderate response to olaparib monotherapy as observed in our experiments. Given the fact that neither BRCA1/2 nor PARP1 exist as homologues in yeast $[45,46]$, we argue that our in silico prediction points not to vulnerability of DNA repair pathways and PARP, but rather to a not yet fully understood mechanism of PARP in regulating cellular growth and mitosis, ultimately targeting proliferative pathways such as PI3K and ERK by involvement in nuclear changes during mitosis, like loosening of chromatin or mitotic spindle assembly [47, 48]. The surprising combinatory effect seen when combined with zoledronic acid should hence encourage the community to evaluate olaparib use in indications aside from DNA-repair deficient tumors.

The synthetic lethal drug combinations identified by our predictor among drugs used in clinical practice all centered about interactions with BCL2 (Table 2). Docetaxel and paclitaxel, primarily targeting tubulin, induce off-target Bcl-2 inhibition by causing permanent Bcl-2 phosphorylation during mitotic arrest $[49,50]$.

Vascular Endothelial Growth Factor (VEGF) has been shown to induce Bcl-2 expression and anti-apoptotic signaling [51-53]. Her2 overexpression has also been found to cause $\mathrm{Bcl}-2$ overexpression and consequent resistance to apoptosis $[54,55]$. In vitro experiments have demonstrated that Bcl-2 expression and stability is regulated by the M2 subunit of the ribonucleotide reductase 1 (RRM1) [56].

Taken together, the synergistic drug combinations found in clinical practice all hint at simultaneous disruptions of interdependent pathways. Among drug combinations found in clinical practice, our predictor identified 23 drug pairs as well as 17 different gene pairs targeting BCL2. The recent success of the Bcl-2 inhibitor venetoclax in lymphoid and myeloid malignancies illustrates how found combinations may be of interest for future experiments $[57,58]$.

Although we did not observe full lethality for cells treated within our experiments as might be expected from the concept of synthetic lethality, we propose that the significant reduction of cell viability seen was caused by activation of synthetic lethal pathways. The lack of fully lethal effects in our study may be caused by insufficient pharmacological blockade of the pharmacological targets examined. Furthermore, an effect described as "synthetic sickness", where joint deletion of two genes would lead to a less fit but viable phenotype, might be the mammalian equivalent phenomenon to synthetic lethality in the genetically less complex yeast species $[13,59]$.

A potential bias inherit to the design of our study is that drug selection for combination treatments relied on one specific target as listed by DrugBank. In other words, multiple targets exist for most compounds, and the target inhibition driving the effects observed in our study cannot be distinguished with absolute certainty. As an example, dependent on the concentration used and according to the DrugBank database, celecoxib will not just inhibit PDPK1
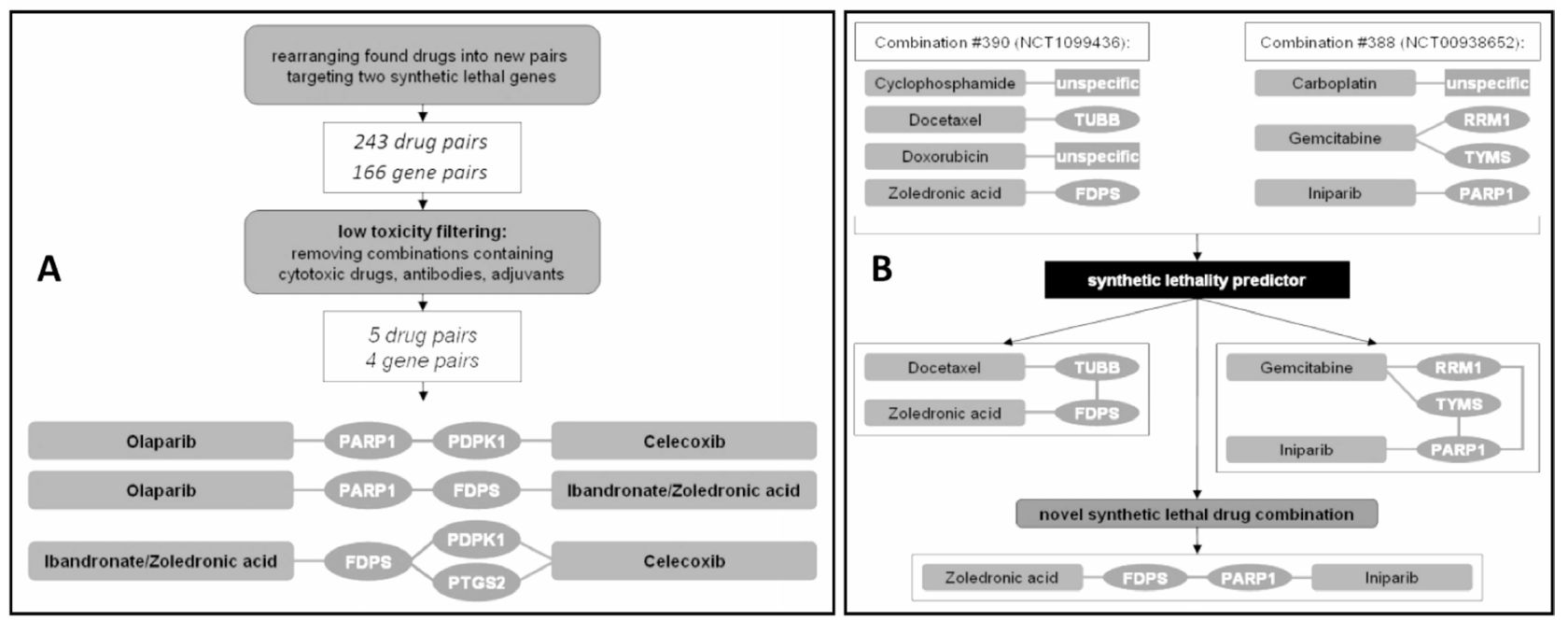

Figure 2: Predicting new drug combinations based on current breast cancer therapy regimens. (A) Of 243 drug pairs covering 166 gene pairs, only 5 drug pairs were found to be non-cytostatic, low-toxicity profile drugs and were further selected for in vitro analysis. (B) In this example, combination \#390 contained the lethal pair docetaxel and zoledronic acid (targeting TUBB and FDPS), while combination \#388 held iniparib and gemcitabine (targeting PARP1 and both RRM1 and TYMS; only predicted drug targets relevant for this figure are depicted for combinations \#388 and \#390). Although not analyzed together in either trial, the combination of iniparib and zoledronic acid was suggested to target a synthetic lethal pair. A list of each drug and gene pair can be found in a Supplementary Dataset 1. 
Table 2: Breast cancer drug combinations used in clinical practice with their supposed synthetic lethal targets

\begin{tabular}{lllc}
\hline & Drug combination & \multicolumn{2}{c}{ Target combination } \\
\hline Drug A & Drug B & Target A & Target B \\
\hline Bevacizumab & Docetaxel & VEGFA & BCL2 \\
Bevacizumab & Paclitaxel & VEGFA & BCL2 \\
Gemcitabine & Docetaxel & RRM1 & BCL2 \\
Gemcitabine & Paclitaxel & RRM1 & BCL2 \\
Trastuzumab & Docetaxel & ERBB2 & BCL2 \\
Trastuzumab & Paclitaxel & ERBB2 & BCL2 \\
\hline
\end{tabular}

Abbreviations: VEGFA: vascular endothelial factor a; BCL2: b-cell lymphoma 2; RRM1: ribonucleotide reductase m1; ERBB22 or Her2, EGFR family receptor 2.

but also six other molecular targets, amongst them the primary target COX-2 (PTGS2) [29, 30]. Although we see the experimental data presented in this article as a proof-of principle of the in silico drug selection approach for synthetic lethal target inhibition, our results might be confounded by such positive molecular "off-target" effects by the compounds themselves, which might only occur upon their combination in vitro. This concern is further augmented by the use of supraphysiological drug concentrations in our study, which are needed to observe meaningful toxicity in vitro. Therefore, we highlight the need for additional experiments with combined gene knockouts of the molecular targets predicted, as well as additional in vivo and clinical studies, to further

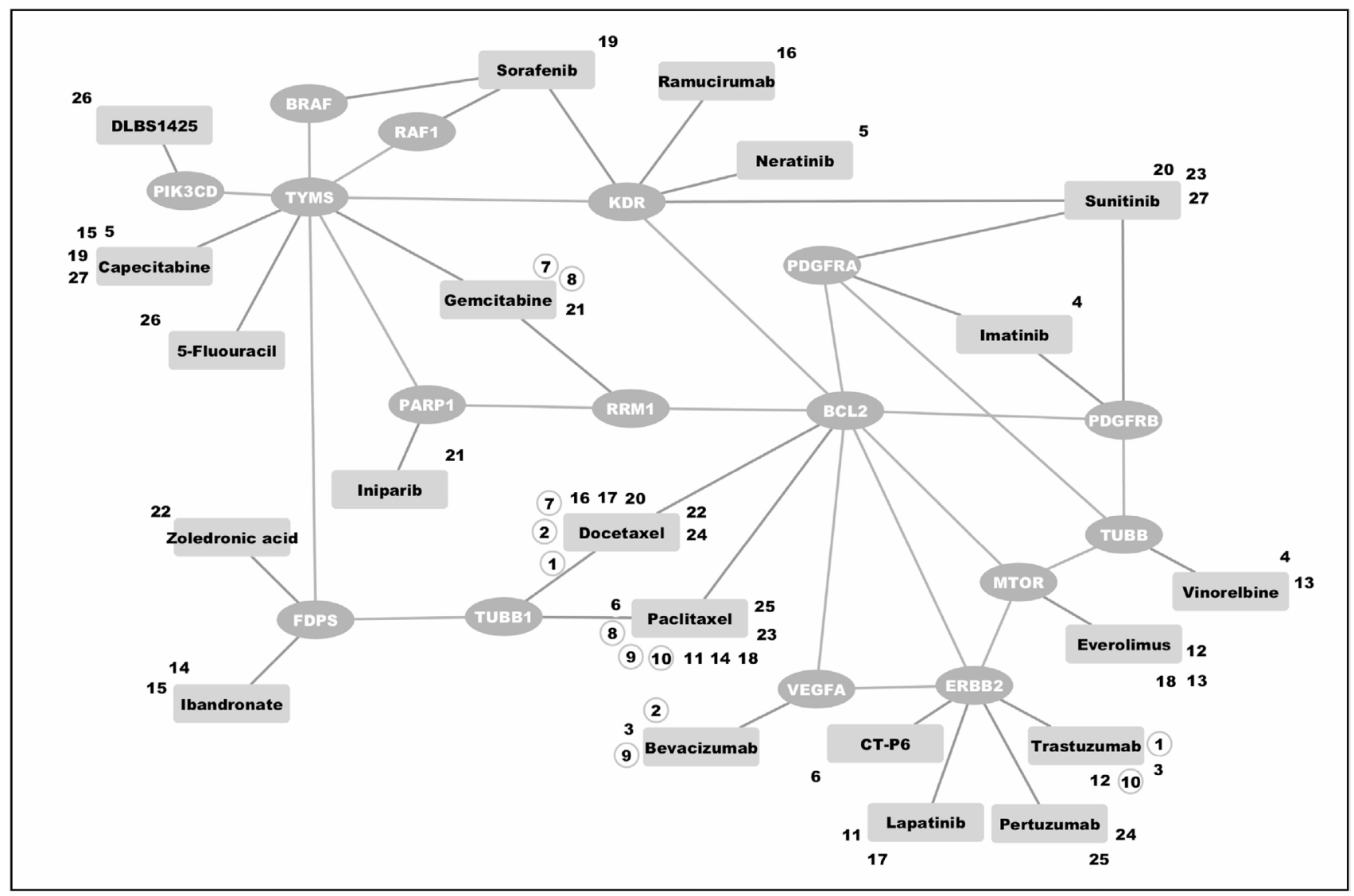

Figure 3: Predicted synthetic lethal interactions among drugs currently used in clinical practice or clinical trials for breast cancer. Drugs found were grouped into pairs covering synthetic lethal gene pairs according to our in silico prediction. Zoledronic acid and docetaxel (as indicated by combination number 22), for instance, may work synergistically by targeting FDPS and TUBB1. Combination numbers in circles link drugs used as combination treatment in clinical practice. A detailed list of drugs and their assigned targets is listed in Supplementary Table 1. 
A

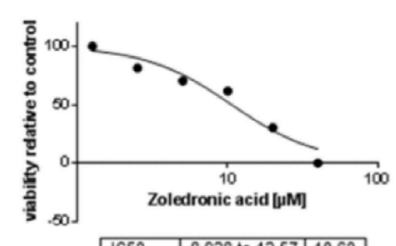

\begin{tabular}{|l|l|l|}
\hline IC50 & 8.928 to 12.57 & 10.60 \\
\hline
\end{tabular}
MDA-MB-468

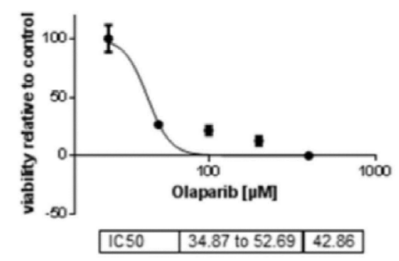

MDA-MB-468
SKBR-3

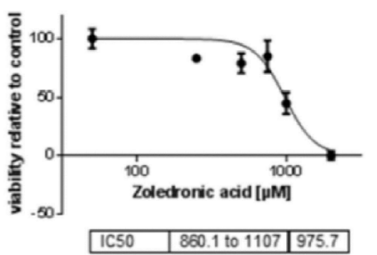

SKBR-3

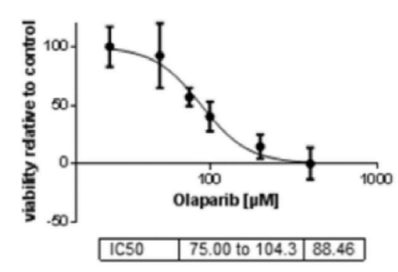

\begin{tabular}{|l|l|l|}
\hline C50 & 75.00 to 104.3 & 88.46 \\
\hline
\end{tabular}

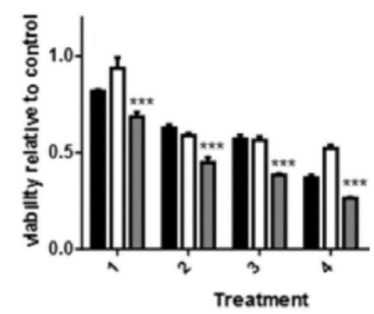

Zoledronic acid 미 Olaparib 口 ZOLOLA
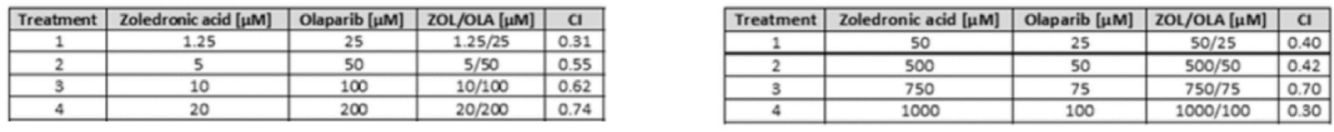

\section{B}

MDA-MB-468
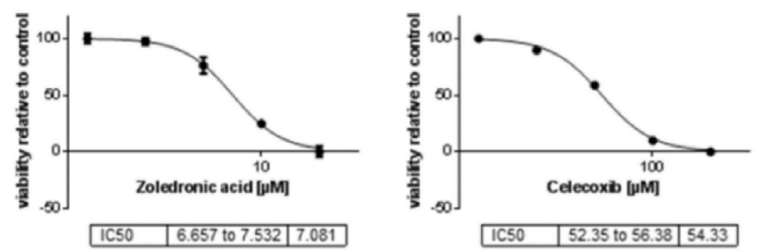

MDA-MB-468
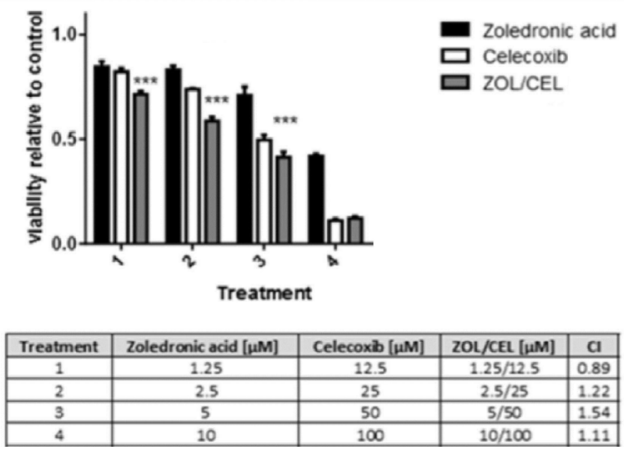

SKBR-3
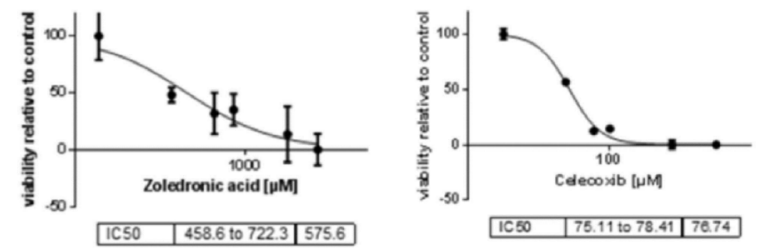

SKBR-3

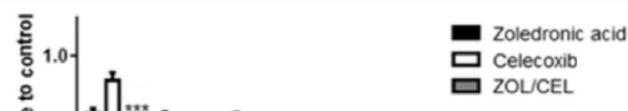
$\square$ Celecoxib Z ZOLCEL

Figure 4: Viability assays of breast cancer cells treated with predicted synthetic lethal drug pairs. Dose-response curves and IC50 values of single agent treatments (upper panels) and viability assay results of combinational treatments (lower panels) of zoledronic acid and olaparib (A, ZOL/OLA) and zoledronic acid and celecoxib (B, ZOL/CEL) in indicated cell lines at increasing drug concentrations. Cells were treated for 48 (A) or 72 hours (B). Combinational treatments with both ZOL/OLA and ZOL/CEL significantly reduced viability in comparison to either single agent treatment. Combinational indices (CI) below 1 (bottom tables) indicate synergism. Bars represent normalized mean values and error bars indicate standard error of the mean (SEM) of three technical replicates. Statistical significance of effects of synergistic treatment compared to single treatments was determined as described in "Materials and Methods" and is indicated by asterisks using adjusted $p$-values $\left({ }^{*} p<0.05,{ }^{* *} p<0.01\right.$ and $\left.{ }^{* * *} p<0.001\right)$. All experiments were performed at least three times, a representative figure is shown. 
evaluate the synthetic lethal effects found through our bioinformatical approach. Also, further studies separately investigating molecular subtypes of breast cancer using a comparable approach are dearly needed to address concerns about potential subtype specificity of our findings.

\section{MATERIALS AND METHODS}

\section{Translation of synthetic lethal gene pairs into human orthologues}

Genetic interactions for Saccharomyces cerevisiae were obtained from DRYGIN, a synthetic genetic array covering more than 5 million gene pair interactions $[60,61]$. For identifying negative genetic interactions (i.e. synthetic lethal protein coding gene pairs), the cutoff for $p$-value and genetic interaction score were set to 0.05 and -0.08 , respectively. Human orthologues of yeast genes were retrieved utilizing "roundup" [62], "oma browser" [63], "ensembl" [64], "inparanoid" [65] and "HomoloGene" [66].

\section{In silico prediction of synthetic lethality gene interactions in human breast cancer}

For expanding the set of synthetic lethal interactions retrieved from yeast, a computational inference method was applied. For building a classifier allowing inference of synthetic lethal interactions beyond given orthologue information from yeast, data including KEGG and PANTHER pathway identifiers [67, 68], Gene Ontology assignment according to PIR slim [69], disease association according to NCBI Medical Subject Headings (MeSH) terms [70] and drug association according to DrugBank (target, enzyme, transporter and carrier associations) was used (https://www.drugbank.ca) [29, 30]. To parameterize synthetic lethal interactions Dice's coefficients for annotation of endpoints, the mean of node-based graph-measures and the shortest path between synthetic lethal nodes were calculated. Subsequently, a machine learning algorithm (random forest model) was created with a training set and validated on a test set of gene interactions, as previously described by our group [71]. For detailed description of predictor generation see Supplementary Figure 1.
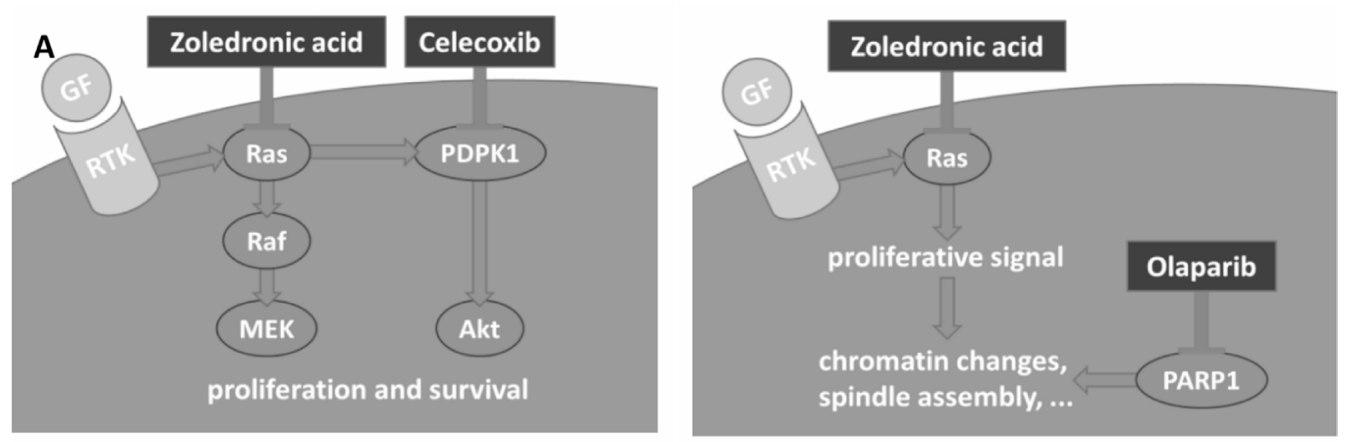

B
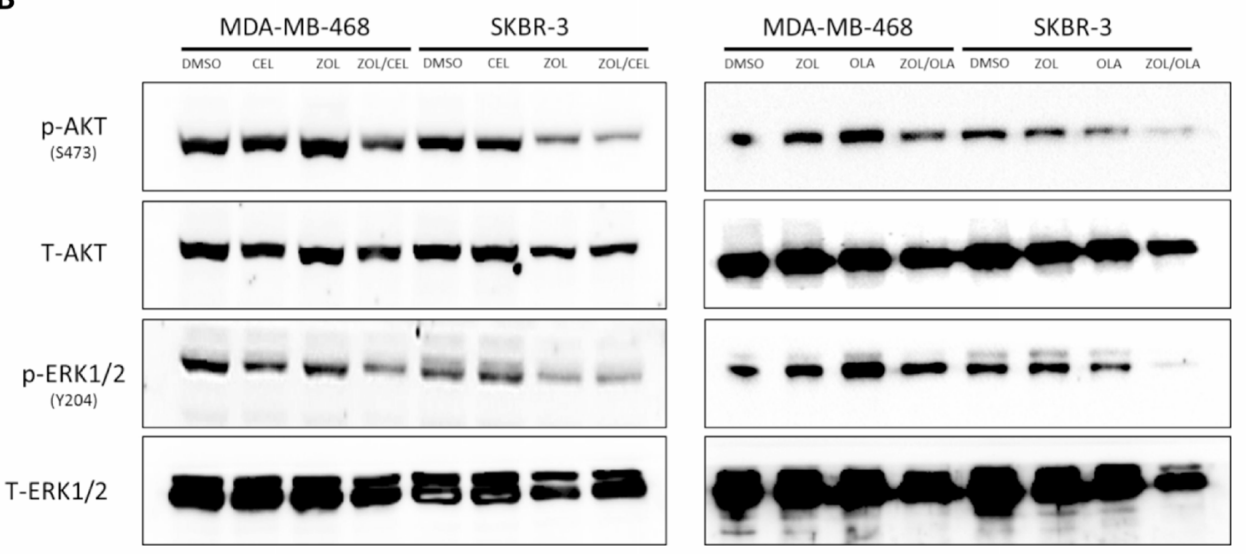

Figure 5: Suggested mechanism of drug interactions found. (A) In silico prediction of synthetic lethality using a yeast-based screen was found for the two drug pairs of zoledronic acid and celcoxib (left) as well as zoledronic acid and olaparib (right). Zoledronic acid inhibits Ras activation by interfering with prenylation. Celecoxib blocks phosphoinositide-dependent kinase-1 (PDPK1), causing disruption of signaling of the Akt pathway. PARP inhibitors disrupt the coordination of chromatin changes and spindle assembly, leading to hindered cell division when combined with zoledronic acid, simultaneously blocking anti-apoptotic signals via Ras inhibition. (B) Western Blot analyses showing disruption of Akt and Erk signaling upon combination treatment of ZOL/CEL (left) and ZOL/OLA (right) in SKBR3 and MDA-MB-468 cells treated at their respective IC50s for 48 hours. Representative blot of three independent experiments is shown. 


\section{Identification of new synergistic drug combinations}

To create new and potentially synergistic drug combinations, data on current pharmacological cancer therapy was collected. Following this step, rearrangement of drugs into new combinations targeting at least one synthetic lethal gene pair based on in silico prediction was performed. Treatment combinations from clinical practice and phase III/IV clinical trial information were obtained from clinical guidelines and "http://clinicaltrials.gov" [72] in August 2012 by searching for the term "cancer". Indications were unified according to MeSH [70]. Drugs from eligible combinations were listed by indication and assigned to their targets using DrugBank. A schematic overview of this process can be seen in Supplementary Figure 2.

\section{Cell culture and reagents}

Human breast cancer cell lines MDA-MB-468 (ATCC ${ }^{\circledR}$ HTB $132^{\mathrm{TM}}$ ) and SKBR-3 (ATCC ${ }^{\circledR}$ HTB-30 ${ }^{\mathrm{TM}}$ ) as well as the human benign mammary epithelial cell line MCF12A (ATCC ${ }^{\circledR}$ CRL-10782 ${ }^{\mathrm{TM}}$ ) were purchased from the American Type Culture Collection (ATCC) and maintained at $37^{\circ} \mathrm{C}$ in a humidified atmosphere with $5 \%$ $\mathrm{CO}_{2}$. ATCC ${ }^{\circledR} \mathrm{HTB}-26^{\mathrm{TM}}$ (MDA-MB-231) cells were kindly provided by Walter Berger, Institute of Cancer Research, Medical University of Vienna. Cells were cultivated in either Dulbecco's Modified Eagle Medium or McCoy's 5a Medium Modified (only SKBR-3), supplemented with $10 \%$ fetal bovine serum and 50 units $/ \mathrm{ml}$ penicillin $\mathrm{G}$, and $50 \mu \mathrm{g} / \mathrm{ml}$ streptomycin sulfate. Celecoxib (CEL) and zoledronic acid (ZOL) were purchased from SigmaAldrich (Sigma, St. Louis, MO, USA). Olaparib (OLA) was provided by AstraZeneca (AstraZeneca, London, United Kingdom). Drugs were diluted in DMSO (CEL and OLA) or PBS (ZOL) respectively.

\section{Viability assays}

$\mathrm{IC}_{50}$ values were determined by performing doseresponse curves by treating cells in a dose escalating manner. Cells were treated in a dose escalating manner. 2500 cells were plated in $50 \mu \mathrm{l}$ of culture medium in 96-well plates overnight. The next day, $50 \mu \mathrm{l}$ of medium containing the double amount of the desired concentration was added and incubated. Cell survival was measured at time points up to 72 hours by using CellTiter-Blue ${ }^{\circledR}$ (Promega, Madison, WI, USA) according to the manufacturers' protocol. Treatments were conducted in triplicates, averaged, and standardized to control (DMSO treatment). All statistical computations were performed using Prism 6 (GraphPad Software, Inc., USA). Multiple group analysis was performed using twoway analysis of variance (ANOVA) and Dunnetts' multiple comparison test as a post-hoc test. Synergy was quantified utilizing CompuSyn and by calculating the combinational index [73].

\section{Immunoblotting}

200.000 cells were cultivated in a 12 -well format and treated with IC50 concentrations for single or combinational treatment for 48 hours. Proteins were isolated using RIPA lysis buffer supplemented with cOmplete Protease Inhibitor Cocktail Tablets (Roche Diagnostics, Mannheim, Germany) and PhosSTOP Phosphatase Inhibitor Cocktail Tablets (Roche Diagnostics). Protein concentration was measured using Bradford reagent (Sigma-Aldrich, St. Louis, Missouri, USA). $30 \mu \mathrm{g}$ of protein was loaded and separated on a $10 \%$ polyacrylamid gel in a Tris-Glycin-SDS buffer. Proteins were then blotted onto nitrocellulose membranes using Turboblot (Biorad, Hercules, California, USA). Membranes were blocked in 5\% bovine serum albumin (Sigma-Aldrich) diluted in TBS-T and incubated with primary antibodies overnight at $4^{\circ} \mathrm{C}$. Primary antibodies used were P-AKT Ser473 (Cell Signaling, \#4060), T-AKT (Cell Signaling, \#), P-ERK1/2 Tyr204 (Santa Cruz, sc-101761), P-ERK1/2 Thr202/Tyr204 (Cell Signaling, \#9101), T-ERK 1/2 (Santa Cruz, sc-93/sc-154). Visualization was performed by using horseradish peroxidase (HRP)-conjugated secondary antibodies (Santa Cruz Laboratories, Dallas, Texas, USA) and the enhanced chemiluminescence detection system (Biorad) on a ChemiDoc XRS+ Imaging device (Biorad).

\section{Annexin/7-AAD stainings}

200.000 cells were seeded into wells of 12 -well plates and left to adhere overnight. Cells were treated at the IC50 of each compound. At indicated time points, cells were then trypsinized and stained with AnnexinV-APC (BD Bioscience, 550474) and 7-AAD (ebioscience, 00-6993-50) according to the manufacturers protocol. Cell fluorescence was captured using a FACS Canto II device (BD Bioscience) and analyzed using FlowJo software (FlowJo LLC, V10).

\section{Abbreviations}

PARP: Poly (ADP-ribose) polymerase; BRCA1/2: Breast Cancer susceptibility gene 1/2; SSR: Single strand repair; HDR: Homology directed repair; RNA: Ribonucleic acid; CRISPR: Clustered regularly interspaced short palindromic repeats; CAS: CRISPR associated; TNBC: Triple Negative Breast Cancer; EMT: Epithelial-Mesenchymal transition; EGFR: Epidermal Growth Factor Receptor; COX-2: Cyclooxygenase 2; PDPK1: Phosphoinositide-dependent kinase-1; FAP: Familial adenomatous polyposis; PAR: Poly(ADP-ribose); DNA: Deoxyribonucleic acid; VEGF: Vascular endothelial growth factor; RRM1: Ribonucleotide reductase 1; IC50: Half maximal inhibitory concentration; ATCC: American Type Culture Collection; CO2: Carbon dioxide; DMSO: Dimethyl sulfoxide; 7-AAD: 7-Aminoactinomycin D. 


\section{Author contributions}

Conception and design: PM, PP, MK, PH, MM; Development of methodology: All authors; Acquisition of data: MM, MS, ET, AH, PP, SU; Manuscript writing: MM, MS, ET; Interpretation of data and manuscript approval: All authors.

\section{ACKNOWLEDGMENTS}

The authors would like to acknowledge Barbara Ziegler, Corinna Altenberger and Gerwin Heller for their tremendous support and collegiality throughout the last years. We would also like to thank David J. Rickles for discussing the manuscript and Maria König for helping with laboratory administration and experimental preparations.

\section{CONFLICTS OF INTEREST}

The authors report no conflicts of interest.

\section{FUNDING}

This work was generously supported by a translational research grant to $\mathrm{MM}$ by the Austrian Society for Hematology and Oncology (OeGHO). ET is recipient of a doctoral fellowship of the Austrian Academy of Sciences (DOC Programme, OeAW). Olaparib was generously provided free of charge through a restricted material transfer agreement by Astra Zeneca.

\section{REFERENCES}

1. Dobzhansky T. Genetics of natural populations; recombination and variability in populations of Drosophila pseudoobscura. Genetics. 1946; 31:290-290.

2. Hartwell LH, Szankasi P, Roberts CJ, Murray AW, Friend $\mathrm{SH}$. Integrating genetic approaches into the discovery of anticancer drugs. Science. 1997; 278:1064-1068.

3. Kaelin WG Jr. The concept of synthetic lethality in the context of anticancer therapy. Nat Rev Cancer. 2005; 5:689-698.

4. Srivas R, Shen JP, Yang CC, Sun SM, Li J, Gross AM, Jensen J, Licon K, Bojorquez-Gomez A, Klepper K, Huang J, Pekin D, Xu JL, et al. A Network of Conserved Synthetic Lethal Interactions for Exploration of Precision Cancer Therapy. Mol Cell. 2016; 63:514-525.

5. Bryant HE, Schultz N, Thomas HD, Parker KM, Flower D, Lopez E, Kyle S, Meuth M, Curtin NJ, Helleday T. Specific killing of BRCA2-deficient tumours with inhibitors of poly(ADP-ribose) polymerase. Nature. 2005; 434:913-917.
6. Farmer H, McCabe N, Lord CJ, Tutt AN, Johnson DA, Richardson TB, Santarosa M, Dillon KJ, Hickson I, Knights C, Martin NM, Jackson SP, Smith GC, Ashworth A. Targeting the DNA repair defect in BRCA mutant cells as a therapeutic strategy. Nature. 2005; 434:917-921.

7. Ashworth A. A synthetic lethal therapeutic approach: poly(ADP) ribose polymerase inhibitors for the treatment of cancers deficient in DNA double-strand break repair. J Clin Oncol. 2008; 26:3785-3790.

8. Gudmundsdottir K, Ashworth A. The roles of BRCA1 and BRCA2 and associated proteins in the maintenance of genomic stability. Oncogene. 2006; 25:5864-5874.

9. Murai J, Huang SY, Das BB, Renaud A, Zhang Y, Doroshow JH, Ji J, Takeda S, Pommier Y. Trapping of PARP1 and PARP2 by Clinical PARP Inhibitors. Cancer Res. 2012; 72:5588-5599.

10. Thompson JM, Nguyen QH, Singh M, Razorenova OV. Approaches to identifying synthetic lethal interactions in cancer. Yale J Biol Med. 2015; 88:145-155.

11. Yu B, Luo J. Synthetic lethal genetic screens in Ras mutant cancers. Enzymes. 2013; 34:201-219.

12. Ruiz S, Mayor-Ruiz C, Lafarga V, Murga M, VegaSendino M, Ortega S, Fernandez-Capetillo O. A Genomewide CRISPR Screen Identifies CDC25A as a Determinant of Sensitivity to ATR Inhibitors. Mol Cell. 2016; 62:307-313.

13. Madhukar NS, Elemento O, Pandey G. Prediction of Genetic Interactions Using Machine Learning and Network Properties. Front Bioeng Biotechnol. 2015; 3:172.

14. Wildenhain J, Spitzer M, Dolma S, Jarvik N, White R, Roy M, Griffiths E, Bellows DS, Wright GD, Tyers M. Prediction of Synergism from Chemical-Genetic Interactions by Machine Learning. Cell Syst. 2015; 1:383-395.

15. McManus KJ, Barrett IJ, Nouhi Y, Hieter P. Specific synthetic lethal killing of RAD54B-deficient human colorectal cancer cells by FEN1 silencing. Proc Natl Acad Sci U S A. 2009; 106:3276-3281.

16. Vizeacoumar FJ, Arnold R, Vizeacoumar FS, Chandrashekhar M, Buzina A, Young JT, Kwan JH, Sayad A, Mero P, Lawo S, Tanaka H, Brown KR, Baryshnikova A, et al. A negative genetic interaction map in isogenic cancer cell lines reveals cancer cell vulnerabilities. Mol Syst Biol. 2013; 9:696.

17. Wu M, Li X, Zhang F, Li X, Kwoh CK, Zheng J. In silico prediction of synthetic lethality by meta-analysis of genetic interactions, functions, and pathways in yeast and human cancer. Cancer Inform. 2014; 13:71-80.

18. Paul JM, Templeton SD, Baharani A, Freywald A, Vizeacoumar FJ. Building high-resolution synthetic lethal networks: a ,Google map ' of the cancer cell. Trends Mol Med. 2014; 20:704-715.

19. Han K, Jeng EE, Hess GT, Morgens DW, Li A, Bassik MC. Synergistic drug combinations for cancer identified in a CRISPR screen for pairwise genetic interactions. Nat Biotechnol. 2017; 35:463-474. 
20. Jerby-Arnon L, Pfetzer N, Waldman YY, McGarry L, James D, Shanks E, Seashore-Ludlow B, Weinstock A, Geiger T, Clemons PA, Gottlieb E, Ruppin E. Predicting cancer-specific vulnerability via data-driven detection of synthetic lethality. Cell. 2014; 158:1199-1209.

21. Ben-Aharon I, Vidal L, Rizel S, Yerushalmi R, Shpilberg O, Sulkes A, Stemmer SM. Bisphosphonates in the adjuvant setting of breast cancer therapy--effect on survival: a systematic review and meta-analysis. PLoS One. 2013; 8:e70044.

22. Eriksen EF, Diez-Perez A, Boonen S. Update on longterm treatment with bisphosphonates for postmenopausal osteoporosis: a systematic review. Bone. 2014; 58:135-135.

23. Gnant M, Mlineritsch B, Schippinger W, LuschinEbengreuth G, Postlberger S, Menzel C, Jakesz R, Seifert M, Hubalek M, Bjelic-Radisic V, Samonigg H, Tausch C, Eidtmann H, et al. Endocrine therapy plus zoledronic acid in premenopausal breast cancer. N Engl J Med. 2009; 360:679-691.

24. Gnant M, Mlineritsch B, Stoeger H, Luschin-Ebengreuth G, Knauer M, Moik M, Jakesz R, Seifert M, Taucher S, Bjelic-Radisic V, Balic M, Eidtmann H, Eiermann W, et al. Zoledronic acid combined with adjuvant endocrine therapy of tamoxifen versus anastrozol plus ovarian function suppression in premenopausal early breast cancer: final analysis of the Austrian Breast and Colorectal Cancer Study Group Trial 12. Ann Oncol. 2015; 26:313-320.

25. Luckman SP, Hughes DE, Coxon FP, Graham R, Russell G, Rogers MJ. Nitrogen-containing bisphosphonates inhibit the mevalonate pathway and prevent post-translational prenylation of GTP-binding proteins, including Ras. J Bone Miner Res. 1998; 13:581-589.

26. Goodsell DS. The molecular perspective: the ras oncogene. Oncologist. 1999; 4:263-264.

27. Neville-Webbe HL, Holen I, Coleman RE. The anti-tumour activity of bisphosphonates. Cancer Treat Rev. 2002; 28:305-319.

28. Senaratne SG, Pirianov G, Mansi JL, Arnett TR, Colston KW. Bisphosphonates induce apoptosis in human breast cancer cell lines. Br J Cancer. 2000; 82:1459-1468.

29. Wishart DS, Feunang YD, Guo AC, Lo EJ, Marcu A, Grant JR, Sajed T, Johnson D, Li C, Sayeeda Z, Assempour N, Iynkkaran I, Liu Y, et al. DrugBank 5.0: a major update to the DrugBank database for 2018. Nucleic Acids Res. 2018; 46:D1074-D1082.

30. Wishart DS, Knox C, Guo AC, Shrivastava S, Hassanali M, Stothard P, Chang Z, Woolsey J. DrugBank: a comprehensive resource for in silico drug discovery and exploration. Nucleic Acids Res. 2006; 34:D668-672.

31. Schech AJ, Kazi AA, Gilani RA, Brodie AH. Zoledronic acid reverses the epithelial-mesenchymal transition and inhibits self-renewal of breast cancer cells through inactivation of NF-kappaB. Mol Cancer Ther. 2013; 12:1356-1366.
32. Subik K, Lee JF, Baxter L, Strzepek T, Costello D, Crowley P, Xing L, Hung MC, Bonfiglio T, Hicks DG, Tang P. The Expression Patterns of ER, PR, HER2, CK5/6, EGFR, Ki-67 and AR by Immunohistochemical Analysis in Breast Cancer Cell Lines. Breast Cancer (Auckl). 2010; 4:41-41.

33. Kroep JR, Charehbili A, Coleman RE, Aft RL, Hasegawa Y, Winter MC, Weilbaecher K, Akazawa K, Hinsley S, Putter H, Liefers GJ, Nortier JW, Kohno N. Effects of neoadjuvant chemotherapy with or without zoledronic acid on pathological response: A meta-analysis of randomised trials. Eur J Cancer. 2016; 54:63-63.

34. Davies NM, McLachlan AJ, Day RO, Williams KM. Clinical pharmacokinetics and pharmacodynamics of celecoxib: a selective cyclo-oxygenase- 2 inhibitor. Clin Pharmacokinet. 2000; 38:225-242.

35. Kulp SK, Yang YT, Hung CC, Chen KF, Lai JP, Tseng PH, Fowble JW, Ward PJ, Chen CS. 3-phosphoinositidedependent protein kinase-1/Akt signaling represents a major cyclooxygenase-2-independent target for celecoxib in prostate cancer cells. Cancer Res. 2004; 64:1444-1451.

36. Zhu J, Huang JW, Tseng PH, Yang YT, Fowble J, Shiau CW, Shaw YJ, Kulp SK, Chen CS. From the cyclooxygenase-2 inhibitor celecoxib to a novel class of 3-phosphoinositidedependent protein kinase-1 inhibitors. Cancer Res. 2004; 64:4309-4318.

37. Morisaki T, Umebayashi M, Kiyota A, Koya N, Tanaka H, Onishi H, Katano M. Combining celecoxib with sorafenib synergistically inhibits hepatocellular carcinoma cells in vitro. Anticancer Res. 2013; 33:1387-1395.

38. Zhang H, Li Z, Wang K. Combining sorafenib with celecoxib synergistically inhibits tumor growth of nonsmall cell lung cancer cells in vitro and in vivo. Oncol Rep. 2014; 31:1954-1960.

39. Steinbach G, Lynch PM, Phillips RK, Wallace MH, Hawk E, Gordon GB, Wakabayashi N, Saunders B, Shen Y, Fujimura T, Su LK, Levin B, Godio L, et al. The effect of celecoxib, a cyclooxygenase-2 inhibitor, in familial adenomatous polyposis. N Engl J Med. 2000; 342:1946-1952.

40. Kaufman B, Shapira-Frommer R, Schmutzler RK, Audeh MW, Friedlander M, Balmana J, Mitchell G, Fried G, Stemmer SM, Hubert A, Rosengarten O, Steiner M, Loman N, et al. Olaparib monotherapy in patients with advanced cancer and a germline BRCA1/2 mutation. J Clin Oncol. 2015; 33:244-250.

41. Lee JM, Ledermann JA, Kohn EC. PARP Inhibitors for BRCA1/2 mutation-associated and BRCA-like malignancies. Ann Oncol. 2014; 25:32-40.

42. Yamamoto N, Nokihara H, Yamada Y, Goto Y, Tanioka M, Shibata T, Yamada K, Asahina H, Kawata T, Shi X, Tamura T. A Phase I, dose-finding and pharmacokinetic study of olaparib (AZD2281) in Japanese patients with advanced solid tumors. Cancer Sci. 2012; 103:504-509. 
43. Helleday $T$. The underlying mechanism for the PARP and BRCA synthetic lethality: clearing up the misunderstandings. Mol Oncol. 2011; 5:387-393.

44. Liu L, Ling X, Tang H, Chen J, Wen Q, Zou F. Poly(ADPribosyl)ation enhances H-RAS protein stability and causes abnormal cell cycle progression in human TK6 lymphoblastoid cells treated with hydroquinone. Chem Biol Interact. 2015; 238:8-8.

45. Claussin C, Chang M. Multiple Rad52-Mediated HomologyDirected Repair Mechanisms Are Required to Prevent Telomere Attrition-Induced Senescence in Saccharomyces cerevisiae. PLoS Genet. 2016; 12:e1006176.

46. Ame JC, Spenlehauer C, de Murcia G. The PARP superfamily. Bioessays. 2004; 26:882-893.

47. Chang P, Jacobson MK, Mitchison TJ. Poly(ADP-ribose) is required for spindle assembly and structure. Nature. 2004; 432:645-649.

48. Kim MY, Zhang T, Kraus WL. Poly(ADP-ribosyl)ation by PARP-1: ,PAR-laying' NAD+ into a nuclear signal. Genes Dev. 2005; 19:1951-1967.

49. Haldar S, Chintapalli J, Croce CM. Taxol induces bcl-2 phosphorylation and death of prostate cancer cells. Cancer Res. 1996; 56:1253-1255.

50. Sakurikar N, Eichhorn JM, Chambers TC. Cyclin-dependent kinase-1 (Cdk1)/cyclin B1 dictates cell fate after mitotic arrest via phosphoregulation of antiapoptotic Bcl-2 proteins. J Biol Chem. 2012; 287:39193-39204.

51. Pidgeon GP, Barr MP, Harmey JH, Foley DA, BouchierHayes DJ. Vascular endothelial growth factor (VEGF) upregulates BCL-2 and inhibits apoptosis in human and murine mammary adenocarcinoma cells. Br J Cancer. 2001; $85: 273-278$.

52. Nor JE, Christensen J, Mooney DJ, Polverini PJ. Vascular endothelial growth factor (VEGF)-mediated angiogenesis is associated with enhanced endothelial cell survival and induction of Bcl-2 expression. Am J Pathol. 1999; 154:375-384.

53. Gerber HP, Dixit V, Ferrara N. Vascular endothelial growth factor induces expression of the antiapoptotic proteins Bcl-2 and A1 in vascular endothelial cells. J Biol Chem. 1998; 273:13313-13316.

54. Kumar R, Mandal M, Lipton A, Harvey H, Thompson CB. Overexpression of HER2 modulates bcl-2, bcl-XL, and tamoxifen-induced apoptosis in human MCF-7 breast cancer cells. Clin Cancer Res. 1996; 2:1215-1219.

55. Zhang GJ, Kimijima I, Onda M, Kanno M, Sato H, Watanabe T, Tsuchiya A, Abe R, Takenoshita S. Tamoxifeninduced apoptosis in breast cancer cells relates to downregulation of bcl-2, but not bax and bcl-X(L), without alteration of p53 protein levels. Clin Cancer Res. 1999; 5:2971-2977.

56. Rahman MA, Amin AR, Wang D, Koenig L, Nannapaneni S, Chen Z, Wang Z, Sica G, Deng X, Chen ZG, Shin DM.
RRM2 regulates Bcl-2 in head and neck and lung cancers: a potential target for cancer therapy. Clin Cancer Res. 2013; 19:3416-3428.

57. Anderson MA, Huang D, Roberts A. Targeting BCL2 for the treatment of lymphoid malignancies. Semin Hematol. 2014; 51:219-227.

58. Roberts AW, Davids MS, Pagel JM, Kahl BS, Puvvada SD, Gerecitano JF, Kipps TJ, Anderson MA, Brown JR, Gressick L, Wong S, Dunbar M, Zhu M, et al. Targeting BCL2 with Venetoclax in Relapsed Chronic Lymphocytic Leukemia. N Engl J Med. 2016; 374:311-322.

59. Bandyopadhyay N, Ranka S, Kahveci T. SSLPred: predicting synthetic sickness lethality. Pac Symp Biocomput. 2012:7-18.

60. Costanzo M, Baryshnikova A, Bellay J, Kim Y, Spear ED, Sevier CS, Ding H, Koh JL, Toufighi K, Mostafavi S, Prinz J, St Onge RP, VanderSluis B, et al. The genetic landscape of a cell. Science. 2010; 327:425-431.

61. Koh JL, Ding H, Costanzo M, Baryshnikova A, Toufighi K, Bader GD, Myers CL, Andrews BJ, Boone C. DRYGIN: a database of quantitative genetic interaction networks in yeast. Nucleic Acids Res. 2010; 38:D502-507.

62. DeLuca TF, Cui J, Jung JY, St Gabriel KC, Wall DP. Roundup 2.0: enabling comparative genomics for over 1800 genomes. Bioinformatics. 2012; 28:715-716.

63. Altenhoff AM, Schneider A, Gonnet GH, Dessimoz C. OMA 2011: orthology inference among 1000 complete genomes. Nucleic Acids Res. 2011; 39:D289-294.

64. Kersey PJ, Allen JE, Christensen M, Davis P, Falin LJ, Grabmueller C, Hughes DS, Humphrey J, Kerhornou A, Khobova J, Langridge N, McDowall MD, Maheswari U, et al. Ensembl Genomes 2013: scaling up access to genomewide data. Nucleic Acids Res. 2014; 42:D546-552.

65. Remm M, Storm CE, Sonnhammer EL. Automatic clustering of orthologs and in-paralogs from pairwise species comparisons. J Mol Biol. 2001; 314:1041-1052.

66. Coordinators NR. Database resources of the National Center for Biotechnology Information. Nucleic Acids Res. 2014; 42:D7-17.

67. Kanehisa M, Goto S, Sato Y, Kawashima M, Furumichi M, Tanabe M. Data, information, knowledge and principle: back to metabolism in KEGG. Nucleic Acids Res. 2014; 42:D199-205.

68. Thomas PD, Campbell MJ, Kejariwal A, Mi H, Karlak B, Daverman R, Diemer K, Muruganujan A, Narechania A. PANTHER: a library of protein families and subfamilies indexed by function. Genome Res. 2003; 13:2129-2141.

69. Hunter S, Jones P, Mitchell A, Apweiler R, Attwood TK, Bateman A, Bernard T, Binns D, Bork P, Burge S, de Castro E, Coggill P, Corbett M, et al. InterPro in 2011: new developments in the family and domain prediction database. Nucleic Acids Res. 2012; 40:D306-312. 
70. Rogers FB. Medical subject headings. Bull Med Libr Assoc. 1963; 51:116-116.

71. Sollner J, Grohmann R, Rapberger R, Perco P, Lukas A, Mayer B. Analysis and prediction of protective continuous B-cell epitopes on pathogen proteins. Immunome Res. 2008; 4:1.
72. McCray AT. Better access to information about clinical trials. Ann Intern Med. 2000; 133:609-614.

73. Chou TC. Drug combination studies and their synergy quantification using the Chou-Talalay method. Cancer Res. 2010; 70:440-446. 